\title{
The evolution of color naming reflects pressure for efficiency: Evidence from the recent past
}

\author{
Noga Zaslavsky, ${ }^{1, *}{ }^{\dagger}$ Karee Garvin,,$^{2}{ }^{\dagger}$ Charles Kemp, ${ }^{3}$ Naftali Tishby, ${ }^{4}$ and Terry Regier $^{2,5}$ \\ ${ }^{1}$ Department of Brain and Cognitive Sciences and Center for Brains Minds and Machines, Massachusetts Institute \\ of Technology, MA 02139, USA, ${ }^{2}$ Department of Linguistics, University of California, Berkeley, CA 94720, USA, \\ ${ }^{3}$ School of Psychological Sciences, University of Melbourne, Victoria 3010, Australia, ${ }^{4}$ Edmond and Lily Safra \\ Center for Brain Sciences and Benin School of Computer Science and Engineering, The Hebrew University of \\ Jerusalem, Jerusalem 9190401, Israel and ${ }^{5}$ Cognitive Science Program, University of California, Berkeley, CA \\ 94720, USA \\ *Corresponding author. nogazs@mit.edu \\ $\dagger$ These authors contributed equally to this work
}

\begin{abstract}
It has been proposed that semantic systems evolve under pressure for efficiency. This hypothesis has so far been supported largely indirectly, by synchronic cross-language comparison, rather than directly by diachronic data. Here, we directly test this hypothesis in the domain of color naming, by analyzing recent diachronic data from Nafaanra, a language of Ghana and Côte d'Ivoire, and comparing it with quantitative predictions derived from the mathematical theory of efficient data compression. We show that color naming in Nafaanra has changed over the past four decades while remaining near-optimally efficient, and that this outcome would be unlikely under a random drift process that maintains structured color categories without pressure for efficiency. To our knowledge, this finding provides the first direct evidence that color naming evolves under pressure for efficiency, supporting the hypothesis that efficiency shapes the evolution of the lexicon.
\end{abstract}

Key words: language evolution; language change; semantic typology; color naming; efficient communication; information theory; compression

\section{Introduction}

What forces shape the evolution of semantic systems (Majid et al., 2015)? This general question has often been addressed in the specific case of color naming. Many theories hold that languages acquire new color terms with time, resulting in increasingly fine-grained color naming systems (Berlin and Kay, 1969; Kay and Maffi, 1999; MacLaury, 1997; Levinson, 2000; but see also Haynie and Bowern, 2016). More recently, it has also been claimed (e.g., Lindsey et al., 2015; Regier et al., 2015; Gibson et al., 2017; Kemp et al., 2018; Zaslavsky et al., 2018; Conway et al., 2020) that this historical evolutionary process, and color naming more generally, are shaped by the need for efficient communication - that is, the need to communicate accurately with a simple lexicon.

However, most research concerning the evolution of color naming has been based indirectly on synchronic cross-language comparison, rather than directly on fine-grained diachronic data collected in the field. There are some approaches that have approximated this ideal: e.g. Biggam (2012) considered historical texts; Kay (1975) considered informant age as a proxy for change over time; and Haynie and Bowern (2016) used phylogenetic methods to infer the history of color naming in a particular language family. However, these remain approximations: historical texts, while providing genuinely diachronic data, do not support analyses at a fine-grained level close to color perception; informant age is a reasonable proxy for change over time, but still a proxy; and phylogenetic reconstruction provides an inferred historical record rather than a directly measured one.

Here, we examine color naming evolution directly, using fine-grained diachronic data from the field for a single language, Nafaanra. We do so in a theory-driven manner, by testing quantitative predictions for language change previously derived from the theoretical framework of Zaslavsky, Kemp, Regier, and Tishby (2018, henceforth ZKRT). This framework integrates the proposal that languages evolve under pressure for efficient communication (Regier et al., 2015; Lindsey et al., 
bioRxiv preprint doi: https://doi.org/10.1101/2021.11.03.467047; this version posted November 16, 2021. The copyright holder for this preprint (which was not certified by peer review) is the author/funder, who has granted bioRxiv a license to 2 display the preprint in perpetuity. It is made available under aCC-BY-NC-ND 4.0 International license.

2015; Gibson et al., 2017; Kemp et al., 2018) together with rate-distortion theory (Shannon, 1959; Berger, 1971), the branch of information theory that characterizes efficient data compression under limited communicative resources.

We find that: (1) color naming in Nafaanra has changed during the recent past by adding new color terms and becoming more semantically fine-grained; (2) this has happened in a way that is consistent with pressure for efficiency; and (3) this outcome would be unlikely under a process of random drift that maintains structured color categories without pressure for efficiency. To our knowledge, this is the first finding that directly supports the proposal that color naming evolves under pressure for efficiency. Xu et al. (2016) previously used a related theoretical framework to show that a specific mechanism of semantic change - semantic chaining - shows signs of pressure for efficiency in a different semantic domain, that of names for containers. Our present work generalizes that earlier finding by showing direct pressure for efficiency in language change that is not restricted to chaining, using a different framework, and in a domain - color naming - for which questions of evolution and language change have long been theoretically central.

In what follows, we first discuss color naming in Nafaanra, comparing data from 1978 with data that one of us (K.G.) collected in 2018. We then review the theoretical framework of ZKRT and test its predictions in the case of semantic evolution in Nafaanra color naming. We conclude by discussing implications of our findings.

\section{Color naming and its evolution in Nafaanra}

Nafaanra is a Senufo language spoken in Ghana and Côte d'Ivoire, with approximately 61,000 speakers across all dialects (Simons and Gordon, 2006). The Nafaanra data in this study were collected in the town of Banda Ahenkro, Ghana. Community members estimate that the greater Banda region currently has around 20,000 speakers of Nafaanra spread throughout the area, with around 6,000 speakers in Banda Ahenkro proper (Garvin, 2017). In Banda Ahenkro, Nafaanra is the most commonly spoken language and is used across all domains. However, within the Banda Ahenkro community, there are no known monolingual speakers of Nafaanra except for small children, as many Nafaanra speakers also speak Twi, a member of the Kwa language family (Simons and Gordon, 2006), and English, to varying degrees of frequency and fluency. Twi serves as a lingua franca beyond Banda Ahenkro, and English is the national language, learned and used in education. Proficiency for Twi is generally higher than for English, and Twi is used

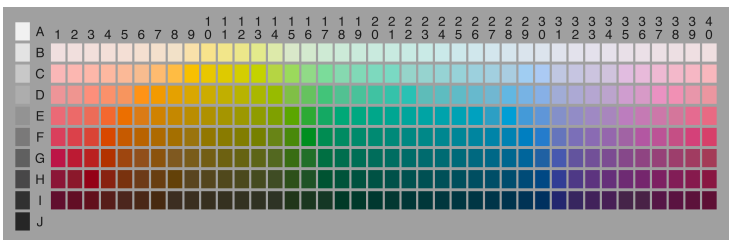

Figure 1. Color naming stimulus grid used in the World Color Survey (WCS). The grid contains 330 stimulus chips: 320 color chips, and 10 achromatic chips shown in the leftmost column. Participants were shown each chip in a fixed pseudo-random order, and asked to name the color of the chip.

more frequently and across more domains. However, media is often in English, and thus, while proficiency in English is lower, exposure to English is still high. Despite the influence of Twi and English, Nafaanra is dominant for Nafaanra speakers in the Banda Ahenkro region. Community members understand the current overall language usage profiles to be comparable between 1978 and 2018 (the two years of data collection), with Nafaanra as the dominant language, and some Twi and English usage in trade and education respectively; however, speakers also report an increase in usage and exposure to Twi and especially English since 1978.

Color naming data for Nafaanra were initially collected in 1978 in Banda Ahenkro, as part of the World Color Survey (WCS; Kay et al. 2009), following WCS protocol. ${ }^{1}$ Participants in the WCS were shown each of the 330 color chips in the color naming grid shown in Figure 1, in a fixed random order, and asked to provide a name for each color.

A total of 29 Nafaanra speakers participated in the 1978 survey, and the resulting data are shown in Figure 2A. The Nafaanra color naming system of 1978 is a 3-term system, with terms for light ('finge'),

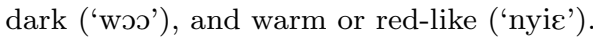

In 2018, 40 years after the original WCS data collection, Nafaanra color naming data were collected again by one of us (K.G.), in the same town, Banda Ahenkro, and following the same protocol. ${ }^{2}$ Speakers were asked to provide a color term for each chip in the stimulus grid ('yga woo yi hin?'; What is the color?). A total of 15 Nafaanra speakers participated in the 2018 study, 6 female and 9

\footnotetext{
1 WCS data are available at http://www.icsi. berkeley.edu/wcs/data.html. WCS protocol is specified in the Instructions to Fieldworkers, available at https://www1.icsi.berkeley.edu/wcs/images/WCS_ instructions-20041018/jpg/border/index.html.

2 For example, following WCS protocol, the 2018 study was conducted on bright days in the shade to ensure chip visibility and data compatibility with the 1978 data. The chips used were the same as those used in 1978, and were presented in the same order.
} 
A. 1978 system

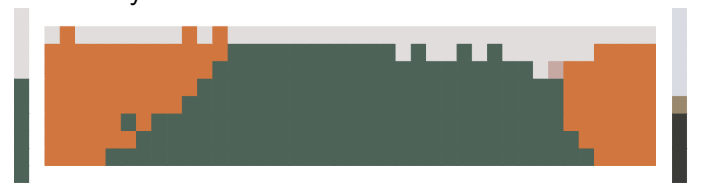

B. 2018 system

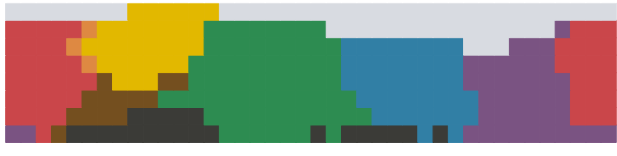

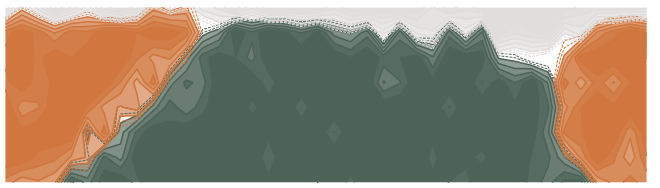

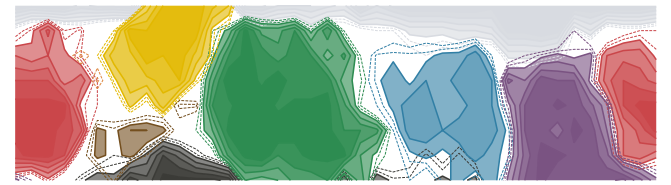

Figure 2. Color naming and its evolution in Nafaanra. The Nafaanra color naming system in 1978 (A) and in 2018 (B), plotted against the color naming grid of Figure 1. Each color term is shown in the color that corresponds to the center of mass of its color category. Mode maps (top) show the modal term for each color chip. Contour plots (bottom) show the proportion of color term use across participants. Dashed lines correspond to agreement levels of $40 \%-45 \%$, and solid lines correspond to agreement

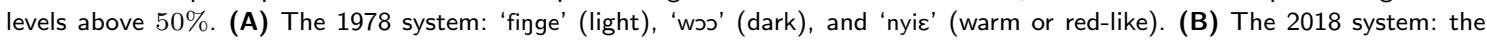
three terms from 1978 have smaller extensions and new terms have emerged — 'wrenyinge' (green), 'lomru' (orange), 'Đgonyina' (yellow-orange), 'mbruku' (blue), 'poto' (purple), 'wrewaa' (brown), and 'toonro' (gray).

male, ranging in age from $18-77 .{ }^{3}$ The resulting data are shown in Figure 2B. The 2018 system contains the same three color terms as the 1978 system: light ('finge'), dark ('woว'), and warm or redlike ('nyiع') — but these now have smaller extensions, and the system also includes seven new color terms: green ('wrenyinge'), orange ('lomru'), yellow-orange ('ngonyina'), blue ('mbruku'), purple ('poto'), brown ('wrewaa'), and gray ('toonr’'). While these terms represent the most common responses, there was also some variability in term usage for a few categories; specifically, a small number of speakers used 'nyanyinge' 4 instead of 'wrenyinge' for green, 'ndemimi' or 'mimi' instead of 'ygonyina' for yelloworange, and 'tra' instead of 'wrewaa' for brown. One additional term, 'grazaan' for red-brown, was used by a single speaker and for a small portion of chips.

As can be seen in Figure 2, the Nafaanra color naming system changed substantially between 1978 and 2018, becoming more semantically finegrained through the addition of new color terms and adjustment in extension of previously existing terms. However, these qualitative observations alone do not determine whether the system has changed in a way that is consistent with pressure for efficiency. To address that question, we turn next to a formal theoretical framework that captures the idea of communicative efficiency and generates

\footnotetext{
3 A pilot round of data collection took place one year earlier, in 2017, following the same procedure. In 2017, data were collected from 10 participants, 6 male and 4 female, ranging in age from 20-68. The 2017 data are qualitatively comparable to the 2018 data.

4 'nyanyinge' only occurs in the 2017 pilot data for a single speaker.
}

precise testable predictions for how color naming may change continuously over time.

\section{Theoretical framework and predictions}

It has been argued that systems of semantic categories are shaped by functional pressure for communicative efficiency (see Kemp et al., 2018, for a review). This general proposal has been explored in the case of color naming (Lindsey et al., 2015; Regier et al., 2015; Gibson et al., 2017; Zaslavsky et al., 2018; Conway et al., 2020), as well as in other semantic domains, such as kinship (Kemp and Regier, 2012), numeral systems (Xu et al., 2020), and indefinite pronouns (Denic et al., 2020). We are interested in testing whether color naming, and semantic systems more generally, change over time while maintaining communicative efficiency.

To this end, we consider the theoretical framework of Zaslavsky et al. (2018, ZKRT), who argued that languages achieve communicative efficiency by compressing meanings into words via the Information Bottleneck (IB) optimization principle (Tishby et al., 1999). This framework is particularly useful in our context for several reasons. First, it is comprehensively grounded in ratedistortion theory (Shannon, 1959; Berger, 1971), the subfield of information theory characterizing efficient data compression under limited resources, offering firm and independently motivated mathematical foundations. Second, it has previously been applied to color naming and was shown to account for much of the known variation across languages, including fine-grained details such as soft category boundaries and patterns of inconsistent naming (Zaslavsky et al., 2018). At the same time, this framework is not specific to color and has also been applied to other 
bioRxiv preprint doi: https://doi.org/10.1101/2021.11.03.467047; this version posted November 16, 2021. The copyright holder for this preprint (which was not certified by peer review) is the author/funder, who has granted bioRxiv a license to 4 display the preprint in perpetuity. It is made available under aCC-BY-NC-ND 4.0 International license.

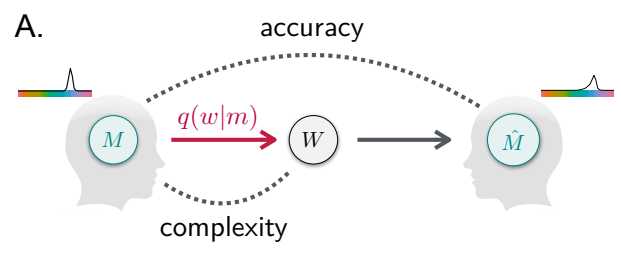

B.

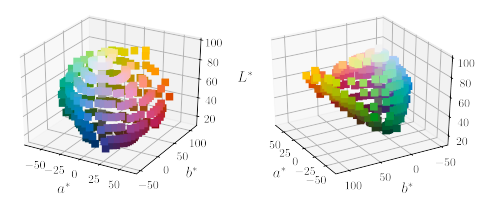

C.

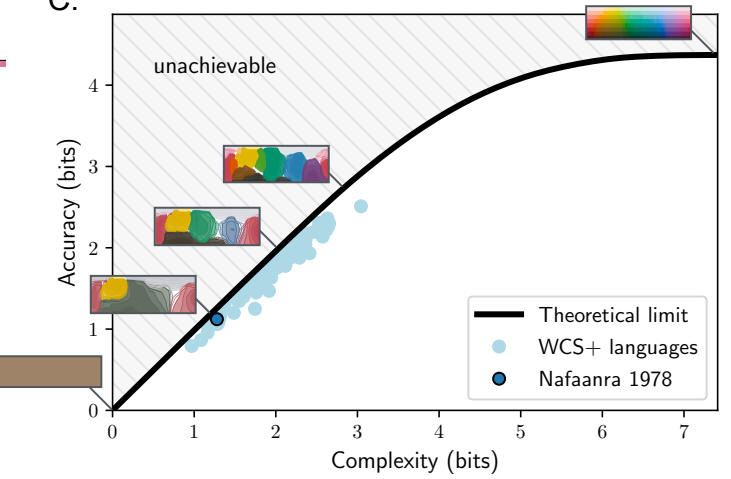

Figure 3. The IB color naming model (adapted from Zaslavsky et al., 2018). A. The communication model described in Section 3.1. B. Two views of the color stimuli of Figure 1 represented in the three perceptual dimensions of the CIELAB space: $L^{*}$ for lightness; $\left(a^{*}, b^{*}\right)$ for hue and saturation in polar coordinates. C. The theoretical limit of efficiency for color naming (black curve) is defined by the set of optimal IB systems for different complexity-accuracy tradeoffs. Contour plots show a few examples of these optimal systems along the curve. Tradeoffs above the curve are unachievable. WCS + languages include the WCS languages and English. Color naming across languages, including in Nafaanra of 1978, is near-optimal.

semantic domains (e.g., Zaslavsky et al., 2019c), suggesting it may characterize the lexicon more broadly.

Third, this framework provides quantitative predictions not only for the efficiency of attested semantic systems, but also for how they may evolve over time and extend beyond those stages already observed. Specifically, this framework suggests an idealized continuous trajectory of semantic evolution in which efficient systems evolve through gradual adjustments of a single complexity-accuracy tradeoff parameter. In the context of color naming, this theoretically-derived evolutionary trajectory was shown by ZKRT to synthesize key aspects of seemingly opposed accounts of color naming evolution (Berlin and Kay, 1969; MacLaury, 1997; Lyons, 1995; Levinson, 2000). This finding suggests that the ZKRT account may explain substantial aspects of language change. However, that possibility has not yet been tested against diachronic data.

Next, we review ZKRT's theoretical framework and its predictions, focusing specifically on its instantiation for color naming which we refer to as the IB color naming model. ${ }^{5}$ In Section 4, we will test the predictions of this model on the diachronic Nafaanra color naming data described in the previous section, and assess whether efficiency can explain semantic change over time in Nafaanra.

\subsection{Communication model}

The theoretical framework we review here is based on a simple communication setting (Figure 3A),

5 The IB color naming model is publicly available at https://github.com/nogazs/ib-color-naming. that can be derived from Shannon's communication model (Shannon, 1948). Here, we focus on the case in which a speaker and a listener communicate about colors, and attention is restricted specifically to the colors shown in Figure 3B, each of which is represented as a point $U$ in a standard perceptual color space, CIELAB. The speaker has a mental representation $M$ of one of these colors $U$, drawn from a prior distribution $p(m){ }^{6}$ This mental representation $M$ is assumed to be a Gaussian distribution in CIELAB space, centered at $U$, capturing the speaker's mental uncertainty about the color. The speaker communicates this representation by encoding it into a word $W$ according to a conditional distribution $q(w \mid m)$, which serves as a stochastic encoder. The listener receives $W$ and attempts to infer from it the speaker's representation $M$ by constructing another representation, $\hat{M}$, that approximates $M$. The listener's inferences are Bayesian with respect to the speaker. ${ }^{7}$

\subsection{The theoretical limit of semantic efficiency}

In this formulation, human semantic systems, such as the Nafaanra color naming systems shown in Figure 2, correspond to encoders $q(w \mid m)$. The IB principle characterizes the set of optimal systems in this setting, which are parametrized by a single

6 We take $p(m)$ to be the prior originally used by ZKRT. See (Zaslavsky et al., 2018, 2019b) for more details about this prior, and (Zaslavsky et al., 2019a) for an evaluation of several alternative priors.

7 This is not an assumption, as it can be derived directly from the IB principle (see Zaslavsky et al., 2018, SI Section 1.2.). 
parameter that controls the tradeoff between the complexity and accuracy of the system. As in ratedistortion theory, complexity is measured by the mutual information between the speaker's mental representation $M$ and word $W$,

$$
I_{q}(M ; W)=\sum_{m, w} p(m) q(w \mid m) \log \frac{q(w \mid m)}{q(w)}
$$

which tightly approximates the number of bits required for communication (Cover and Thomas, 2006). Accuracy corresponds to the similarity between the speaker's and listener's representations, and is measured by $I_{q}(W ; U)$. Maximizing this second informational term amounts to minimizing the expected Kullback-Leibler (KL) divergence between $M$ and $\hat{M} .^{8}$ Thus, high accuracy implies that the listener's inferred representation is similar to the speaker's representation.

Achieving high accuracy requires a complex lexicon, while reducing complexity may result in accuracy loss. According to the IB principle, optimal systems minimize complexity while maximizing accuracy for some tradeoff $\beta \geq 0$ between these two competing objectives. Formally, an optimal encoder $q(w \mid m)$ for a given value of $\beta$ is one that attains the minimum of the IB objective function,

$$
\mathcal{F}_{\beta}[q]=I_{q}(M ; W)-\beta I_{q}(W ; U),
$$

across all possible encoders. Let $\mathcal{F}_{\beta}^{*}$ be the minimal value of this objective for a given value of $\beta$. The theoretical limit of efficiency, also known as the IB curve, is then determined by the set of encoders $q_{\beta}(w \mid m)$ that attain $\mathcal{F}_{\beta}^{*}$ for different values of $\beta$. This limit in the case of color communication is shown by the black curve in Figure 3C, accompanied by a few examples of optimal encoders along the curve.

\subsection{Evolution of the optimal systems}

Intuitively, the tradeoff parameter $\beta$ controls the relative importance of minimizing accuracy over maximizing complexity, and thus how fine-grained a semantic system is. For $\beta \leq 1$, complexity is more important than accuracy, yielding at the optimum a minimally complex yet non-informative system that can be implemented with a single word. This system lies at the origin of the IB curve, as can be seen in Figure 3C. As $\beta$ gradually increases from 1 to $\infty$, the optimal systems evolve in an annealing process along the IB curve, becoming more complex and more accurate. In general, the optimal systems can also change via reverse-annealing, i.e., when $\beta$ gradually decreases, in which case they will travel

8 For a detailed derivation see (Zaslavsky et al., 2018) and (Harremoës and Tishby, 2007). down the curve and become less complex. Along this continuous trajectory, the optimal systems undergo a sequence of structural phase transitions at critical values of $\beta$, in which the number of categories effectively changes (Zaslavsky, 2020).

In the domain of color naming, this theoretical evolutionary trajectory was previously derived from the IB color naming model shown in Figure 3. By mapping the color naming systems of 111 languages (WCS+ dataset) - 110 from the WCS and American English from Lindsey and Brown (2014) — onto optimal systems along this trajectory, it was shown that all of these languages are near-optimal in the IB sense, and that much of the observed crosslanguage variation can be explained by varying $\beta$ alone. Furthermore, it was shown that the optimal trajectory synthesizes aspects of seemingly opposing accounts of color naming evolution. Berlin and Kay's (1969) discrete evolutionary sequence is largely captured by the structural phase transitions that occur at critical points along the trajectory. However, this trajectory is continuous, categories change gradually with $\beta$, and new ones typically emerge in regions of color space that are inconsistently named. These phenomena resonate with approaches to the evolution of color naming (MacLaury, 1997; Lyons, 1995; Levinson, 2000) that traditionally appeared to challenge Berlin and Kay's (1969) proposal.

As noted by ZKRT, these findings suggest that semantic systems, and color naming in particular, evolve under pressure to remain near the IB theoretical limit and that the optimal evolutionary trajectory, while idealized, may capture substantial aspects of language change. From this perspective, the relative importance of accuracy versus complexity, captured by $\beta$, may change over time, driving a system up or down along the theoretical limit, but leaving it near-optimal. Thus, this model makes testable predictions for language change.

\subsection{Quantitative predictions}

We adopt the quantitative predictions and evaluation methods derived by ZKRT, and extend them by explicitly considering the dimension of time. If human semantic systems evolve under pressure to be efficient, i.e., to reach the optimum of (2), then the following two properties should hold over time.

Near-optimality. For each language $l$ with system $q_{l}^{t}(w \mid m)$ at time $t$, there should be a tradeoff $\beta_{l}(t)$ for which the system is near-optimal. Formally, this means that its deviation from optimality,

$$
\varepsilon_{l}(t)=\frac{1}{\beta_{l}(t)}\left(\mathcal{F}_{\beta_{l}(t)}\left[q_{l}^{t}\right]-\mathcal{F}_{\beta_{l}(t)}^{*}\right),
$$

should be small. Because we do not know the true tradeoff parameter, we consider the candidate 


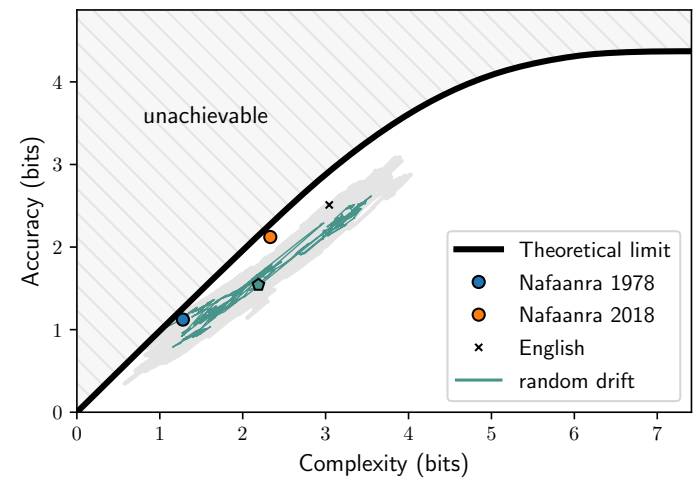

Figure 4. Diachronic efficiency analysis. Color naming in Nafaanra has changed from 1978 to 2018 by climbing up the IB theoretical limit (black curve, same as in Figure 3C), while not reaching the same tradeoff as the English color naming system (data from Lindsey and Brown, 2014). The gray area below the curve shows the area covered by 50 hypothetical trajectories traced out by a process of random drift, which were all initialized near the 1978 system. The green trajectory corresponds to the example of Figure 9 in Appendix $B$, and the pentagon marks its location after 1,500 iterations.

that maps each system to the nearest point along the theoretical limit, i.e., we take $\beta_{l}(t)=$ $\operatorname{argmin}_{\beta}\left\{\mathcal{F}_{\beta}\left[q_{l}^{t}\right]-\mathcal{F}_{\beta}^{*}\right\}$. The system $q_{l}^{t}$ is then taken to be efficient to the extent that $\varepsilon_{l}(t)$ is small, and this can be assessed with respect to counterfactual data, as described below in Section 4.

Structural similarity. Considering $\varepsilon_{l}(t)$ alone reduces the system to only two features - its complexity and its accuracy. However, IB also generates predictions for the full probabilistic structure of $q_{l}^{t}(w \mid m)$. That is, we expect that the full structure of $q_{l}^{t}$ will be similar to that of an optimal system. For simplicity, we compare $q_{l}^{t}$ with $q_{\beta_{l}(t)}$, the optimal system at $\beta_{l}(t)$, but note that it is in principle possible that optimal systems at other values of $\beta$ could be more structurally similar to $q_{l}^{t}$. To measure the structural similarity between two probabilistic category systems, we use the generalized Normalized Information Distance (gNID: Zaslavsky et al., 2018) which was designed for this purpose. That is, $q_{l}^{t}$ and $q_{\beta_{l}(t)}$ are similar to each other to the extent that the gNID between them is small. In this case as well, we will assess the degree of similarity $(1-$ gNID) relative to counterfactual data.

\section{Efficiency and language change}

The previous work reviewed in Section 3 moved from a synchronic efficiency analysis based on crosslanguage data to a diachronic hypothesis that language change is shaped by pressure for efficiency. That diachronic hypothesis has not yet been directly
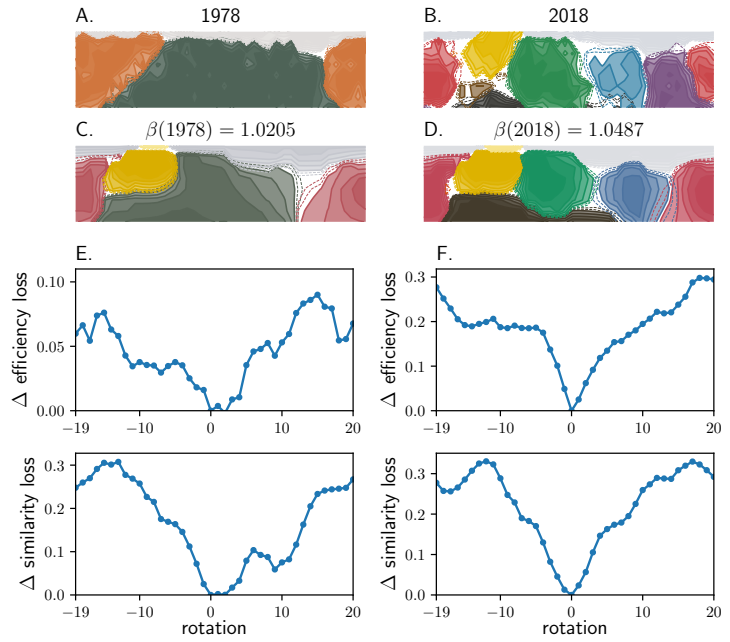

Figure 5. (A-B) Empirical data for Nafaanra in 1978 and 2018 (same as Figure 2). (C-D) Optimal IB systems corresponding to the actual 1978 and 2018 systems. (E-F) Rotation analysis for the 1978 and 2018 Nafaanra systems respectively. $\Delta$ efficiency/similarity loss corresponds to the difference between the score of the rotated and actual system (positive values correspond to higher losses of the rotated system).

tested using fine-grained diachronic data, and the Nafaanra data reported above allow us to fill that gap.

\subsection{Efficiency over time}

First, we are interested in testing whether the efficiency of the Nafaanra color naming system has persisted over time. Because the 1978 Nafaanra data were part of the WCS, we already know from ZKRT's analyses that the 1978 Nafaanra data lay near the limit of efficiency. We conducted an entirely analogous analysis on the 2018 Nafaanra data. Figure 4 shows that the complexity and accuracy of both the 1978 and the 2018 Nafaanra systems are near the theoretical bound, but at different places along the curve. Thus, these diachronic data from Nafaanra appear to be consistent with the near-optimality prediction. Figures 5A-D compare these two natural systems with their corresponding optimal systems that lie directly on the IB curve. It can be seen that the optimal systems capture substantial aspects of the empirical data, but also differ from those data in some respects. For example, the 1978 system lacks a yellow category that is found in the corresponding optimal system, and the 2018 system has purple and brown categories, while the corresponding optimal system does not. While the early yellow category seems to represent a genuine discrepancy between the model and data, the absence of purple and brown does not necessarily. 
bioRxiv preprint doi: https://doi.org/10.1101/2021.11.03.467047; this version posted November 16, 2021. The copyright
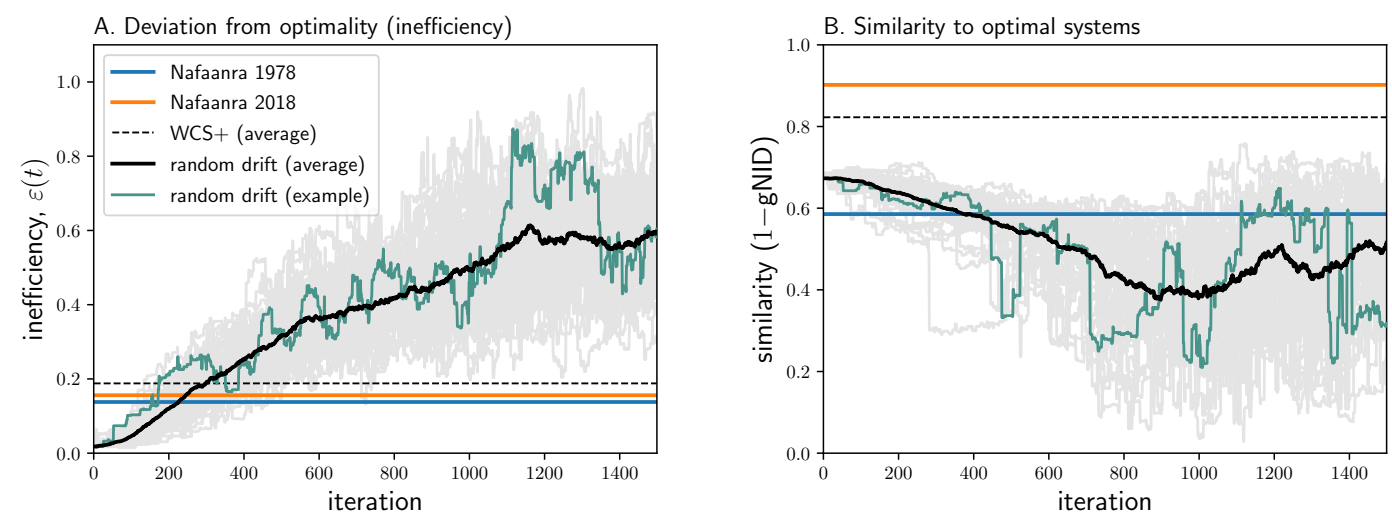

Figure 6. Random drift. (A) Deviation from optimality under random drift (lower values are better). Gray curves correspond to 50 hypothetical trajectories. The green trajectory corresponds to the example of Appendix B, Figure 9 , and the black curve is the average across all 50 trajectories. Nafaanra's deviation from optimality is shown by the blue (1978) and orange (2018) horizontal lines. The black dashed horizontal line shows the average deviation from optimality across the WCS+ languages. (B) Analogous plot showing the structural similarity (higher values are better) with respect to the corresponding optimal system along the IB curve.

These categories emerge at a slightly higher value of $\beta$ (see for example Figure 3C), and therefore this mismatch between the model and data may stem simply from noise in our estimation of $\beta$.

To quantitatively test the extent to which our predictions hold, we evaluated the efficiency loss $\left(\varepsilon_{l}\right)$ and similarity loss (gNID) of the 1978 and 2018 systems, and assessed each system with respect to a set of hypothetical variants. These variants were obtained by rotation in the hue dimension (columns of the WCS stimulus grid; Regier et al., 2007) as illustrated in Appendix A, Figure 8. Following ZKRT, in this analysis $\beta$ was fitted to each system separately in order to consider the best scores these hypothetical systems can achieve. Consistent with ZKRT's findings for the WCS+ languages, including the 1978 Nafaanra system (Figure 5E), the actual (unrotated) 2018 Nafaanra system scores better than any of its hypothetical variants on both measures (Figure 5F). This suggests that the 1978 and 2018 are locally optimal within their set of hypothetical variants, and thus non-trivially efficient. In addition, it can be seen by looking ahead to Figure 6A that these two systems do not deviate much from optimality (less than 0.2 bits), comparable to the average deviation across the WCS+ languages. These results show that 1978 and 2018 Nafaanra are near-optimally efficient when assessed by the same standards that ZKRT used for other color naming systems.

\subsection{Random drift}

So far, we have seen that over the past several decades, the Nafaanra color naming system has changed substantially, while remaining near the theoretical limit of efficiency. This outcome is consistent with our hypothesis that language change may be shaped by functional pressure for efficiency. But before reaching that conclusion, we need to consider a natural alternative: that the same outcome could have been produced by a process of random drift, without any pressure for efficiency. The importance of considering a null model of random drift has recently been emphasized in the literature (e.g. Newberry et al., 2017; Bentz et al., 2018; Karjus et al., 2020), and so here we ask whether a process of random drift could have produced the 2018 Nafaanra system from the 1978 system.

We considered a process of random drift that is described in detail in Appendix B. To avoid random systems, which form a weak baseline, this process maintains some reasonable category structure by representing a color naming system in terms of a set of Gaussian distributions over CIELAB space. It then evolves in a stochastic process that allows existing categories to drift, new categories to emerge, and old categories to occasionally vanish. We generated a set of 50 random drift trajectories, in each case simulating this process for 1, 500 iterations. The initial system was the same for all trajectories, and was obtained by fitting to 1978 Nafaanra, yielding a good approximation of the 1978 system.

The green trajectory in Figure 4 corresponds to one such random drift trajectory, illustrated in Appendix B, Figure 9. The gray area below the IB curve in Figure 4 shows the area traced out by all 50 hypothetical random drift trajectories. It can be seen that these trajectories tend to diverge away from the IB curve, and none reaches the 2018 
Nafaanra system. Figure 6A plots the inefficiency $\left(\varepsilon_{l}\right)$ of the systems in these random drift trajectories over time, and confirms that they tend to become less efficient with time. Interestingly, the same plot also shows that the starting point for these trajectories - a Gaussian approximation to the 1978 Nafaanra system — is more efficient than the 1978 Nafaanra system itself. This demonstrates that the model at the heart of this random drift process can in principle represent highly efficient systems. At the same time, however, the process does not tend to remain at such systems. Figure $6 \mathrm{~B}$ analogously plots the structural similarity between each system in these trajectories on the one hand, and the corresponding optimal system on the other. It can be seen that the random drift process tends to lead to systems that are dissimilar from those along the theoretical efficiency limit. Given these inefficiency and dissimilarity results, it seems unlikely that this process of random drift could have produced the 2018 Nafaanra system, starting from the 1978 system.

\section{Discussion}

The starting point for this study was the claim that systems of semantic categories evolve under functional pressure for efficiency. This claim is consistent with a substantial amount of synchronic data, but it had not previously been tested directly, by bringing it into contact with fine-grained diachronic data that documents language change over time. The present study has addressed that open issue, by considering the evolution of color naming in Nafaanra over the past several decades, through the lens of efficiency.

We have seen that color naming in Nafaanra has changed substantially while remaining nearoptimally efficient, as predicted by the Information Bottleneck (IB) optimality principle and the theory of compression more generally. We have also seen that this outcome would be unlikely under a process of random drift that maintains structured categories but does not incorporate pressure for efficiency. Thus, in at least one language, in at least one semantic domain, and over at least one stretch of time, it appears that a semantic system has evolved in a way that reflects functional pressure for efficiency. To the extent that these results generalize to other languages, domains, and periods of time, they suggest that fundamental informationtheoretic principles may induce a fitness criterion in an evolutionary view of language, guiding the ways in which systems of semantic categories change.

These findings converge with those of a complementary line of work. In a comment on the finding that systems of semantic categories tend to be efficient, Levinson (2012) asked "where our categories come from" - i.e. what process gives rise to these efficient category systems. He suggested that some insight into this question might be obtained from studies of iterated learning that simulate language evolution in the lab (e.g. Kirby et al., 2008; $\mathrm{Xu}$ et al., 2013). This suggestion inspired Carstensen et al. (2015) to explore whether simulated language evolution in the lab in fact produces systems of increasing efficiency. They found that it does, and more recent work has probed this outcome more closely (Carr et al., 2018). Although these earlier studies were based on different formulations of the notion of efficiency, the present work resonates with their findings by showing that actual language change, not just simulated language change, tends toward communicatively efficient semantic systems.

At the same time, the present findings leave a number of points open, some of which suggest directions for future research. We have considered a specific model of random drift for category systems, and while we believe this model to be a reasonable one, it is conceivable that other models of drift could yield different results. More fundamentally, although we have spoken of language evolving under pressure for efficiency, and although our findings are consistent with that idea, we do not know the shape of the trajectory that took Nafaanra from where it was in 1978 to where it was in 2018. The evolution we have seen could have come about in a series of small incremental changes, tracing the IB curve closely, or the system could have been pulled fairly far away from efficiency by some external force, such as language contact, and then gradually retreated to efficiency.

Language contact is an especially relevant consideration in the case of Nafaanra, given the exposure of Nafaanra speakers to English and Twi, as noted above. While it is not known to what extent the evolution in Nafaanra color naming is attributable to contact, it does seem plausible that some of the new 2018 Nafaanra categories may have been borrowed. For example the word 'mbruku' (blue) may plausibly be a borrowing from English 'blue'. However, not all of the new categories show the same evidence of having been borrowed. For example 'ygonyina' (yellow-orange) is Nafaanra for chicken fat, a reasonable description of the color involved; thus the form of this color term does not suggest borrowing. Moreover, although the 2018 Nafaanra system is similar to that of English, it is not a simple copy of it: the category pink is missing, the category orange is minimal and only barely visible in the contour plot of Figure 2B, and the 2018 system has retained the three named categories of the 1978 system, with the same names but with adjusted extensions. Thus, even if substantial parts of the 2018 Nafaanra system were either borrowed from or motivated by English, the Nafaanra categories 
appear to have adjusted their extensions - so some "naturalization" process will have occurred, even in the extreme hypothetical case of largescale borrowing. Further work will be needed to more fully ascertain the role of borrowing, and, to the extent possible, the details of the historical trajectory of Nafaanra language change relative to the theoretical limit. However, whatever the details of that trajectory, our current results based on the beginning and end points of that trajectory do suggest a process that is in some way constrained to either remain, or eventually return to, near the theoretical limit of efficiency.

Nafaanra was one of the first languages in the World Color Survey (WCS) for which color naming data were obtained (Paul Kay, personal communication). Although the WCS data were collected in the 1970 s, the data were only digitized and web-posted in the early 2000s, and they are currently a widely used data resource. It therefore came as a bit of a surprise to us when we realized that these data are actually old enough to be of some historical interest. This realization, and the follow-up work on Nafaanra reported here, opens the possibility of analogous follow-up studies for any or all the 109 other languages in the WCS, to more comprehensively test the hypothesis explored here: that color naming evolves under pressure for efficiency.

\section{Acknowledgments}

We thank Paul Kay for helpful discussions, the Nafaanra community for their help in collecting the data, and Phoebe Killick and Hsin-Yeh Tsai for their help in digitizing the raw data. We also thank Delwin Lindsey and Angela Brown for kindly sharing their English color naming data with us. This study was partially supported by DTRA grant HDTRA11710042 (T.R.), Robert L. Oswalt Graduate Student Support Endowment for Endangered Language Documentation (K.G.), a BCS Fellowship in Computation (N.Z.), and ARC grant FT190100200 (C.K.).

\section{References}

J. T. Abbott, T. L. Griffiths, and T. Regier. Focal colors across languages are representative members of color categories. Proceedings of the National Academy of Sciences, 113(40):11178-11183, 2016. doi: 10.1073/ pnas.1513298113.

C. Bentz, D. Dediu, A. Verkerk, and G. Jäger. The evolution of language families is shaped by the environment beyond neutral drift. Nature Human Behaviour, 2:816-821, 2018.

T. Berger. Rate distortion theory; a mathematical basis for data compression. Prentice-Hall, Englewood Cliffs, NJ, 1971.
B. Berlin and P. Kay. Basic Color Terms: Their Universality and Evolution. University of California Press, Berkeley and Los Angeles, 1969.

C. P. Biggam. The semantics of colour: A historical approach. Cambridge University Press, Cambridge, UK, 2012.

J. W. Carr, K. Smith, J. Culbertson, and S. Kirby. Simplicity and informativeness in semantic category systems. PsyArXiv, 2018.

A. Carstensen, J. Xu, C. T. Smith, and T. Regier. Language evolution in the lab tends toward informative communication. In Proceedings of the 37th Annual Meeting of the Cognitive Science Society, Austin, TX, 2015. Cognitive Science Society.

B. R. Conway, S. Ratnasingam, J. Jara-Ettinger, R. Futrell, and E. Gibson. Communication efficiency of color naming across languages provides a new framework for the evolution of color terms. Cognition, 195:104086, 2020. doi: https://doi.org/ 10.1016/j.cognition.2019.104086.

T. Cover and J. Thomas. Elements of Information Theory (2nd Edition). Wiley-Interscience, Hoboken, NJ, 2006.

M. Denic, S. Steinert-Threlkeld, and J. Szymanik. Complexity/informativeness trade-off in the domain of indefinite pronouns. In Proceedings of the 30th Semantics and Linguistic Theory Conference, 2020.

K. Garvin. Nafaanra documentation project. Number 2017-11. Survey of California and Other Indian Languages, University of California, Berkeley, 2017. doi: 10.7297/X2V98672. With consultants Job Kwabena Ababio, James Anane, Sampson Kwasi Attah, Charles Munufie.

E. Gibson, R. Futrell, J. Jara-Ettinger, K. Mahowald, L. Bergen, S. Ratnasingam, M. Gibson, S. T. Piantadosi, and B. R. Conway. Color naming across languages reflects color use. Proceedings of the National Academy of Sciences, 114(40):1078510790, 2017.

P. Harremoës and N. Tishby. The Information Bottleneck revisited or how to choose a good distortion measure. In IEEE International Symposium on Information Theory, pages 566-571, June 2007.

H. J. Haynie and C. Bowern. Phylogenetic approach to the evolution of color term systems. Proceedings of the National Academy of Sciences, 113(48):1366613671, 2016. doi: 10.1073/pnas.1613666113.

A. Karjus, R. A. Blythe, S. Kirby, and K. Smith. Challenges in detecting evolutionary forces in language change using diachronic corpora. Glossa: A Journal of General Linguistics, 5:45, 2020.

P. Kay. Synchronic variability and diachronic change in basic color terms. Language in Society, 4(3):257270, 1975.

P. Kay and L. Maffi. Color appearance and the emergence and evolution of basic color lexicons. American Anthropologist, 101(4):743-760, 1999.

P. Kay, B. Berlin, L. Maffi, W. R. Merrifield, and R. Cook. The World Color Survey. Stanford: Center for the Study of Language and Information, 2009.

C. Kemp and T. Regier. Kinship categories across languages reflect general communicative principles. Science, 336(6084):1049-1054, 2012. ISSN 00368075 . 
C. Kemp, Y. Xu, and T. Regier. Semantic typology and efficient communication. Annual Review of Linguistics, 4(1), 2018. doi: 10.1146/ annurev-linguistics-011817-045406.

S. Kirby, H. Cornish, and K. Smith. Cumulative cultural evolution in the laboratory: An experimental approach to the origins of structure in human language. Proceedings of the National Academy of Sciences, 105(31):10681-10686, 2008.

S. C. Levinson. Yélî Dnye and the theory of basic color terms. Journal of Linguistic Anthropology, 10(1): $3-55,2000$.

S. C. Levinson. Kinship and human thought. Science, 336(6084):988-989, 2012.

D. T. Lindsey and A. M. Brown. The color lexicon of American English. Journal of Vision, 14(2):17, 2014.

D. T. Lindsey, A. M. Brown, D. H. Brainard, and C. L. Apicella. Hunter-gatherer color naming provides new insight into the evolution of color terms. Current Biology, 25(18):2441-2446, 2015.

J. Lyons. Colour in language. In T. Lamb and J. Bourrieau, editors, Colour: Art and science, pages 194-224. University of Cambridge, Cambridge, UK, 1995.

R. E. MacLaury. Color and cognition in Mesoamerica: Constructing categories as vantages. University of Texas Press, 1997.

A. Majid, F. Jordan, and M. Dunn. Semantic systems in closely related languages. Language Sciences, 49: $1-18,2015$.

M. G. Newberry, C. A. Ahern, R. Clark, and J. B. Plotkin. Detecting evolutionary forces in language change. Nature, 551:223-226, 2017.

T. Regier, P. Kay, and N. Khetarpal. Color naming reflects optimal partitions of color space. Proceedings of the National Academy of Sciences, 104(4):14361441, 2007.

T. Regier, C. Kemp, and P. Kay. Word meanings across languages support efficient communication. In B. MacWhinney and W. O'Grady, editors, The Handbook of Language Emergence, pages 237-263. Wiley-Blackwell, Hoboken, NJ, 2015.

C. E. Shannon. A mathematical theory of communication. Bell System Technical Journal, 27, 1948.
C. E. Shannon. Coding theorems for a discrete source with a fidelity criterion. IRE Nat. Conv. Rec, 4 (142-163):1, 1959.

G. F. Simons and R. G. Gordon. Ethnologue. Encyclopedia of Language and Linguistics, pages $250-253,2006$.

N. Tishby, F. C. Pereira, and W. Bialek. The Information Bottleneck method. In Proceedings of the 37th Annual Allerton Conference on Communication, Control and Computing, 1999.

J. $\mathrm{Xu}, \mathrm{M}$. Dowman, and T. L. Griffiths. Cultural transmission results in convergence towards colour term universals. Proceedings of the Royal Society of London B: Biological Sciences, 280(1758), 2013. doi: $10.1098 / \mathrm{rspb} .2012 .3073$.

Y. Xu, T. Regier, and B. C. Malt. Historical semantic chaining and efficient communication: The case of container names. Cognitive Science, 40(8):20812094, 2016. ISSN 1551-6709. doi: 10.1111/cogs. 12312 .

Y. Xu, E. Liu, and T. Regier. Numeral systems across languages support efficient communication: From approximate numerosity to recursion. Open Mind, 4:57-70, 2020.

N. Zaslavsky. Information-Theoretic Principles in the Evolution of Semantic Systems. Ph.D. Thesis, The Hebrew University of Jerusalem, 2020.

N. Zaslavsky, C. Kemp, T. Regier, and N. Tishby. Efficient compression in color naming and its evolution. Proceedings of the National Academy of Sciences, 115(31):7937-7942, 2018.

N. Zaslavsky, C. Kemp, N. Tishby, and T. Regier. Communicative need in color naming. Cognitive Neuropsychology, 2019a. doi: 10.1080/02643294. 2019.1604502.

N. Zaslavsky, C. Kemp, N. Tishby, and T. Regier. Color naming reflects both perceptual structure and communicative need. Topics in Cognitive Science, 11(1):207-219, 2019b. doi: 10.1111/tops.12395.

N. Zaslavsky, T. Regier, N. Tishby, and C. Kemp. Semantic categories of artifacts and animals reflect efficient coding. In Proceedings of the 41st Annual Meeting of the Cognitive Science Society, pages 1254-1260, 2019c. 
bioRxiv preprint doi: https://doi.org/10.1101/2021.11.03.467047; this version posted November 16, 2021. The copyright holder for this preprint (which was not certified by peer review) is the author/funder, who has granted bioRxiv a license to display the preprint in perpetuity. It is made available under aCC-BY-NC-ND 4.0 International license.

\section{Appendix A. Nafaanra 2018 individual maps}

To provide a complete view of the 2018 Nafaanra color naming data, we present here the color naming map for each participant in the data (Figure 7).

Participant 1

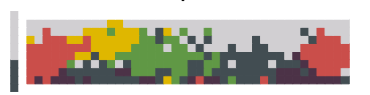

Participant 3

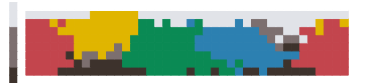

Participant 5

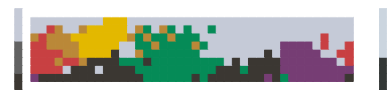

Participant 7

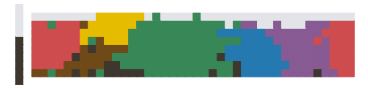

Participant 9

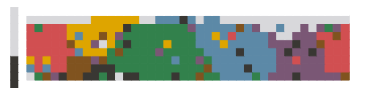

Participant 11

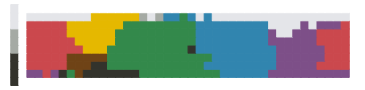

Participant 13

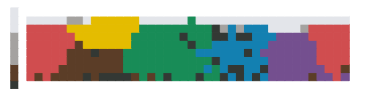

Participant 15

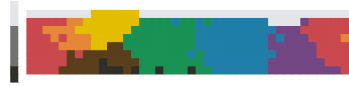

Figure 7. Color naming maps for each participant in the 2018 Nafaanra color naming data. Each chip in the stimulus grid (Figure 1 ) is colored according to its term. The color associated with each term is the color centroid of the term's color category, evaluated per participant.

\section{Appendix B. Rotation analysis}

Our evaluation of the efficiency of the Nafaanra color naming system with respect to a set of hypothetical systems is based on Regier et al.'s (2007) rotation analysis. That is, for each color naming system, a set of hypothetical systems can be derived by rotations along the hue dimension of the WCS color naming grid (Figure 1). This is illustrated in Figure 8 for the 2018 Nafaanra system.
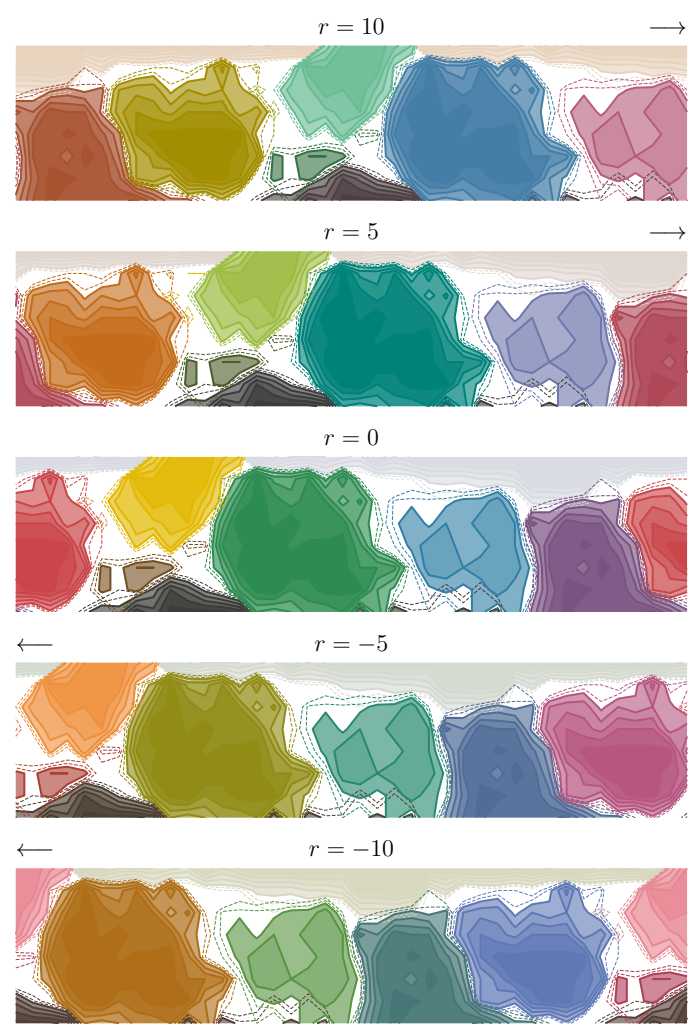

Figure 8. Example of rotated variants of the 2018 Nafaanra system. $r=0$ corresponds to the actual system, $r>0$ to a shift of $r$ columns to the right with respect to the color grid of Figure 1 , and $r<0$ to a shift of $|r|$ columns to the left. 
bioRxiv preprint doi: https://doi.org/10.1101/2021.11.03.467047; this version posted November 16, 2021. The copyright holder for this preprint (which was not certified by peer review) is the author/funder, who has granted bioRxiv a license to 12 display the preprint in perpetuity. It is made available under aCC-BY-NC-ND 4.0 International license.

\section{Appendix C. Random drift model}

Our random drift model simulates language change via a stochastic process that preserves structured categories without incorporating pressure for efficiency. To this end, we consider a class of artificial color naming systems, in which each category $w$ induces a Gaussian distribution, $q(c \mid w)=\mathcal{N}\left(c ; \mu_{w}, \Sigma_{w}\right)$, over CIELAB space (Abbott et al., 2016). In practice, we discretized these Gaussians by restricting them to colors of the WCS grid (Figure 1). A system with $k$ categories is defined by $k$ Gaussians, and a $k$-dimensional probability vector $q(w)$. Given these parameters, the naming distribution is taken to be $q(w \mid c) \propto q(c \mid w) q(w)$, where $c$ is a color. Our stochastic process takes an initial system from this class, and propagates it in time by allowing its parameters to change gradually.

Before we define the dynamics of this process, our parameterization requires further elaboration. First, to ensure that each covariance matrix $\Sigma_{w}$ remains positive semi-definite, we parameterize it by another matrix, $L_{w}$, such that $\Sigma_{w}=L_{w} L_{w}^{\top}$. Second, to allow categories to emerge or vanish, we assume a maximum of $K=330$ potential categories, and keep a weight vector, $\pi_{w}$, for them. Only categories for which $\pi_{w}$ is higher than a given threshold $\eta$ are included in the lexicon. For those categories, we define $q(w) \propto \pi(w)$. Therefore, $\eta$ is a hyperparameter that controls the tendency to add new categories. At the $t$-th iteration of the process, the system is defined by $\theta(t)=\left\{\mu_{w}^{(t)}, L_{w}^{(t)}, \pi_{w}^{(t)}\right\}_{w=1}^{K}$.

Given an initial system, $\theta(0)$, the dynamics of the process are defined as follows. At each iteration $t$, a category $w_{t}$ is chosen at random. First, the weight vector is updated by randomly selecting whether to add or subtract $\eta$ from $\pi_{w_{t}}^{(t-1)}$, and keeping the vector non-negative and normalized. Next, if $w_{t}$ is already in the lexicon, i.e. $\pi_{w_{t}}^{(t-1)}>\eta$, then with probability 0.5 its parameters are updated as follows:

$$
\begin{aligned}
\mu_{w_{t}}^{(t)} & =\frac{1}{2}\left(\mu_{w_{t}}^{(t-1)}+c_{t}\right), \quad c_{t} \sim q_{t-1}\left(c \mid w_{t}\right) \\
L_{w_{t}}^{(t)} & =L_{w_{t}}^{(t-1)}+I+A^{(t)}, \quad A_{i, j}^{(t)} \sim \mathcal{N}(0,1) .
\end{aligned}
$$

The update rule for $\mu_{w_{t}}^{(t)}$ shifts it in the direction of $c_{t}$, which on average would be a small shift because $c_{t}$ is sampled from $q_{t-1}\left(c \mid w_{t}\right)$. The update rule for $L_{w_{t}}^{(t)}$ adds to it a noise matrix, $A^{(t)}$, and the identity matrix, $I$, in order to encourage the category to grow over time.

Finally, it remains to set the initial set of parameters, $\theta(0)$, and threshold $\eta$. We set $\theta(0)$ such that the corresponding system will approximate the iteration 1

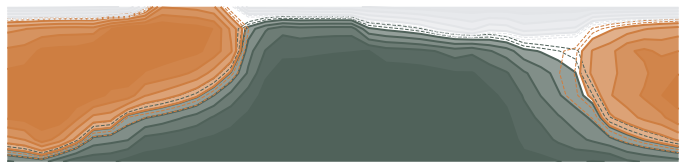

iteration 100

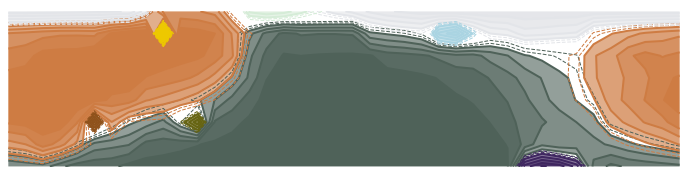

iteration 700

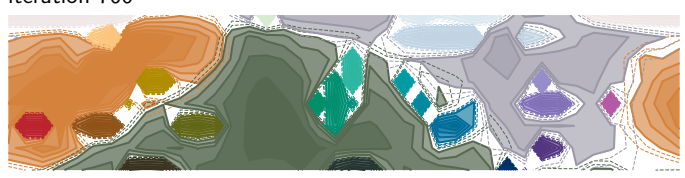

iteration 800

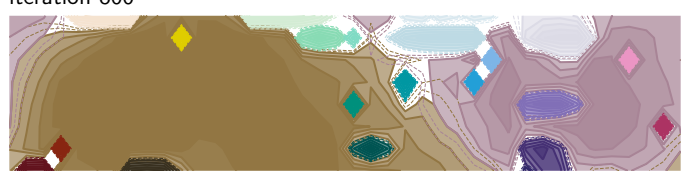

iteration 1500

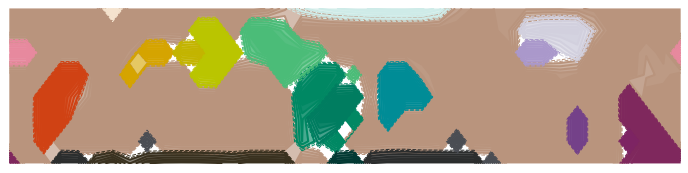

Figure 9. An example of a hypothetical trajectory generated by a process of random random drift. The trajectory was initialized at a system fitted to the 1978 Nafaanra system, and traced out for 1,500 iterations.

actual 1978 Nafaanra system. For each category $w$ in the 1978 system, we fit a Gaussian with a diagonal covariance matrix to that category, and take $L_{w}^{(0)}$ to be its square root. For these categories, we take $\pi_{w}^{(0)}$ to be their proportion in the 1978 naming data. For the remaining potential categories, which are not in the lexicon: we set $\pi_{w}^{(0)}=0$, initialize $\mu_{w}^{(0)}$ by randomly selecting a chip from the WCS grid (with replacement), and initialize $L_{w}^{(0)}$ by $\sigma_{w}^{(0)} I+A_{w}^{(0)}$, where $A_{w}^{(0)} \sim \mathcal{N}(0,1)$ and $\sigma_{w}^{(0)}$ is drawn uniformly from $[1,5]$. We take $\eta=0.01$, for which we observed a trend of gradual increase in the number of categories, reaching on average $k=23.9$ after 1,500 iterations. An example of a hypothetical trajectory that was generated by this random drift process is shown in Figure 9. 